\title{
Application of 3D Acquisition Design Technology in HCX Gas Storage Project*
}

\author{
Jun Wu, Jiangli Chen, Liu Yang, Pandeng Liu, Botao Wang, Wei Liu, Yiwei Li, Xinhua Zhang \\ BGP Southwest Geophysical Branch, CNPC, Chengdu, China \\ Email: wujun_wt@cnpc.com.cn
}

How to cite this paper: $\mathrm{Wu}, \mathrm{J} ., \mathrm{Chen}, \mathrm{J} . \mathrm{L}$., Yang, L., Liu, P.D., Wang, B. T., Liu, W., Li, Y. W., \& Zhang, X. H. (2021). Application of 3D Acquisition Design Technology in HCX Gas Storage Project. Journal of Geoscience and Environment Protection, 9, 176-181.

https://doi.org/10.4236/gep.2021.95013

Received: February 20, 2021

Accepted: May 28, 2021

Published: May 31, 2021

\begin{abstract}
In view of the shallow buried depth of the target layer and it is difficult to obtain high-quality seismic data of HCX gas storage project, based on the acquisition parameters of the adjacent area, the $3 \mathrm{D}$ acquisition technical scheme is designed for focusing on the imaging of the top of HCX high-steep anticline. By optimizing the acquisition parameters and the excitation and receiving conditions, the acquisition of high-resolution and high signal-to-noise ratio seismic data is guaranteed to meet the demand of fracture prediction and reservoir prediction.
\end{abstract}

\section{Keywords}

Gas Storage, High-Steep Anticline, Imaging, Acquisition Parameters

\section{Introduction}

The HCX gas storage project is located in Changshou and Fuling districts of Chongqing, which is the $3 \mathrm{D}$ seismic exploration project for gas storage deployed by Petro China Southwest Oil \& Gasfield Company. The purpose is to further implement the responsibility of gas storage, better support the construction of gas storage, meet the peak shaving and supply guarantee in Sichuan and Chongqing in the future, and take into account the seasonal peak shaving demand of the whole country. The purpose of HCX project deployment is to implement the structural details and fault sealing, the thickness of direct cap rock and bottom supporting layer, the reservoir distribution of $T_{1} j^{2}$ and $T_{1} j^{2}{ }_{1}-T_{1} j^{1}$, and to provide evidence for the overall design of gas storage and the deployment of injection and production wells through the implementation of 3D seismic.

As a gas storage project, HCX project requires high resolution and signal${ }^{\star} \mathrm{HCX}$ gas storage project. 
to-noise ratio of field seismic data. Taking $\mathrm{T}_{1} \mathrm{j}$ of Lower Triassic as the main target layer, in order to obtain seismic reflection data from Jurassic to Silurian and ensure high signal-to-noise ratio data of main target layers, the project team carried out reasonable $3 \mathrm{D}$ acquisition technical scheme design for the imaging of the top of HCX high-steep anticline. By optimizing acquisition parameters and selecting excitation and receiving conditions, the resolution and signal-to-noise ratio of the data are improved.

\section{Seismogeological Characteristics of the Target Area}

\subsection{Surface Seismogeological Conditions}

HCX area is a typical mountainous-hilly landform with an altitude of $180 \mathrm{~m}$ $760 \mathrm{~m}$. In the middle part, there is a long and narrow mountain range in the direction of $\mathrm{NE}$ and $\mathrm{SW}$, with an altitude of $400 \mathrm{~m}-760 \mathrm{~m}$; in the both limbs, there are hills, with an altitude of $180 \mathrm{~m}-450 \mathrm{~m}$ and relatively small fluctuation.

Quartz sandstone of $\mathrm{T}_{3} \mathrm{x}$ and sandstone and mudstone of $\mathrm{J}_{2} \mathrm{~s}$ and $\mathrm{J}_{1}$ are mainly exposed on the surface. More than $88 \%$ of the strata in the area are Jurassic strata, and the excitation and receiving conditions are relatively good.

There are a few surface control points in the $3 \mathrm{D}$ area, the results from the surface of the adjacent area show that: The surface structure of this area is two-layer structure, and the thickness of low velocity layer is between $2 \mathrm{~m}$ and $8 \mathrm{~m}$; the velocity of the high velocity layer is affected by the lithology of the surface. The velocity of the high velocity layer in Jurassic strata is generally between $2200 \mathrm{~m} / \mathrm{s}$ and $3000 \mathrm{~m} / \mathrm{s}$; the velocity of high velocity layer in Triassic strata is about 3000 $\mathrm{m} / \mathrm{s}$, as shown in Figure 1.

\subsection{Deep Seismogeological Conditions}

The East high point of HCX structure is an asymmetric anticline with steep southeast flank and gentle northwest flank, and the southeast flank is cut by C(2) fault. The general structure shape of the shallow layer (above $T_{3} x^{1}$ ) is basically consistent with the surface structure, with simple structure shape and undeveloped faults. The anticline structure in the middle layer $\left(\mathrm{T}_{1} \mathrm{j}_{2}{ }_{2}-\mathrm{P}_{1} \mathrm{l}\right)$ is divided into several fault blocks by faults. The time window of the target layer is in the range of $500 \mathrm{~ms}-1500 \mathrm{~ms}$.
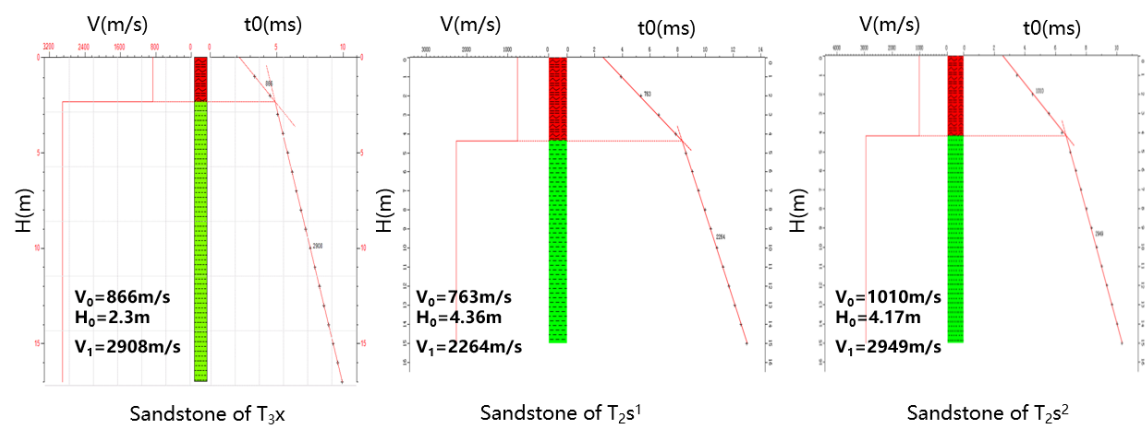

Figure 1. Interpretation of surface control points in adjacent area. 
Four large faults are developed in the East high point of HCX structure, which are all reverse faults. They are mostly developed in Permian and Triassic vertically and distributed in the both limbs of the structure. The strike is nearly NE, which is consistent with the axial direction of the structure, and most of them disappear with the dip of both ends of the structure.

$T_{1} j$ is the main target layer of HCX project. Its buried depth is relatively shallow, ranging from $940 \mathrm{~m}$ to $2900 \mathrm{~m}$, and the high part of the main structure is $940 \mathrm{~m}$ to $1600 \mathrm{~m}$.

The synthetic well records show that the reflection characteristics of the standard layer in the target layer are obvious, which is the basis for obtaining highquality seismic data. In order to predict $\mathrm{T}_{1} \mathrm{j}_{2}{ }_{2}$ thin reservoir $(6 \mathrm{~m}-22 \mathrm{~m})$ according to geological requirements, the frequency bandwidth of seismic data in the target layer should be more than $10 \mathrm{~Hz}-60 \mathrm{~Hz}$.

\section{Design of 3D Acquisition Technical Scheme}

\subsection{Analysis of Old Data}

Twice 2D seismic exploration, twice 3D gas reservoirs and twice 3D reef exploration have been completed in HCX area and adjacent areas. The parameters of the four times $3 \mathrm{D}$ geometry are obviously different. The bin of the $3 \mathrm{D}$ gas storage project is $20 \mathrm{~m} \times 20 \mathrm{~m}$, the line spacing is not more than $240 \mathrm{~m}$, and the maximum offset is about $4000 \mathrm{~m}$.

\subsection{Analysis of the Key and Difficulties}

Based on the comprehensive analysis of geological tasks, working area conditions and actual data, it is concluded that this $3 \mathrm{D}$ exploration is faced with two key and difficulties. The first is to improve the seismic reflection energy, signal-to-noise ratio and longitudinal resolution of $T_{1} j$ to ensure high-fidelity data to meet the needs of fracture prediction and reservoir prediction. The second is to ensure the high precision imaging of $T_{1} j$, which lays a foundation for the prediction of high quality reservoir and small fault fractures.

\subsection{Demonstration of Acquisition Method}

According to the key and difficulties, the project has formulated targeted technical ideas. First, fine surface structure investigation, system excitation parameter test, and supporting high sensitivity single point geophone reception, in order to improve the signal-to-noise ratio of single shot data. Second, to improve the imaging quality of shallow and steeply dipping interface, small bin and small line spacing are adopted. Thirdly, the geometry with high density and high folds is adopted to ensure the imaging effect of complex structures. Fourthly, wideazimuth geometry acquisition and processing is used to characterize the anisotropy of geological bodies to meet the needs of reservoir prediction (Han, J. W., Zhang, W., Zhang, H., Xiao, H., \& Geng, C., 2020). 


\subsection{Demonstration of Acquisition Parameters}

According to the regional drilling situation, four points in different parts of the structure were selected to build geophysical models, and then the acquisition parameters were demonstrated. On the premise of satisfying the requirement of longitudinal resolution, the parameters such as the maximum protection frequency, bin size, folds, the maximum offset, the receiving line distance and the offset aperture are demonstrated successively (Zhang, J. H., 2017). In the parameter demonstration, various factors should be fully considered (Zhang, W. C., 2017). For example, the selection of the maximum offset considers the buried depth of the target layer, the dynamic correction tensile distortion, the accuracy of velocity analysis, the stability of reflection coefficient, the convergence of diffraction energy, and the AVO effect (Guo, W., 2017). In addition, it is better to combine the illumination analysis of the model (Li, W. W., 2008) and the analysis of the actual data of the adjacent areas.

Figure 2 is the illumination analysis results of observation systems with different offsets based on the $2 \mathrm{D}$ geological model established by the depth profile of old data. As can be seen from the figure, after the offset of $4000 \mathrm{~m}$, the growth trend of illumination energy of $\mathrm{TT}_{1}$ f slows down.

Figure 3 and Figure 4 are comparative analysis diagrams of illumination curves at different structural positions and different offsets. After the offset reaches $4000 \mathrm{~m}$, the increase range of illumination energy curves of $\mathrm{TT}_{1} \mathrm{j}_{2}{ }_{2}$ and $\mathrm{TT}_{1} \mathrm{f}$ is small. Therefore, the maximum offset is recommended at about 4000 $\mathrm{m}$.

\section{Analysis of Application Effect}

In this exploration, small receiving line spacing, high folds, shot-receiver density,
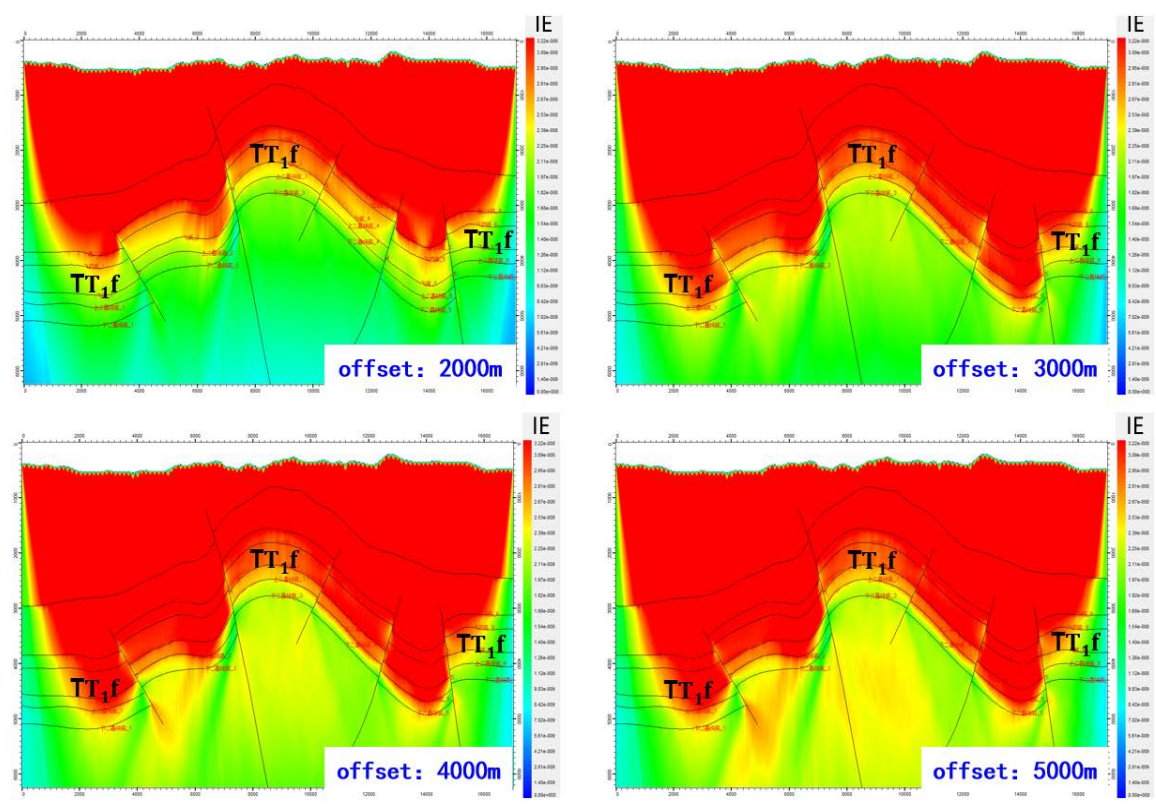

Figure 2. Illumination analysis. 

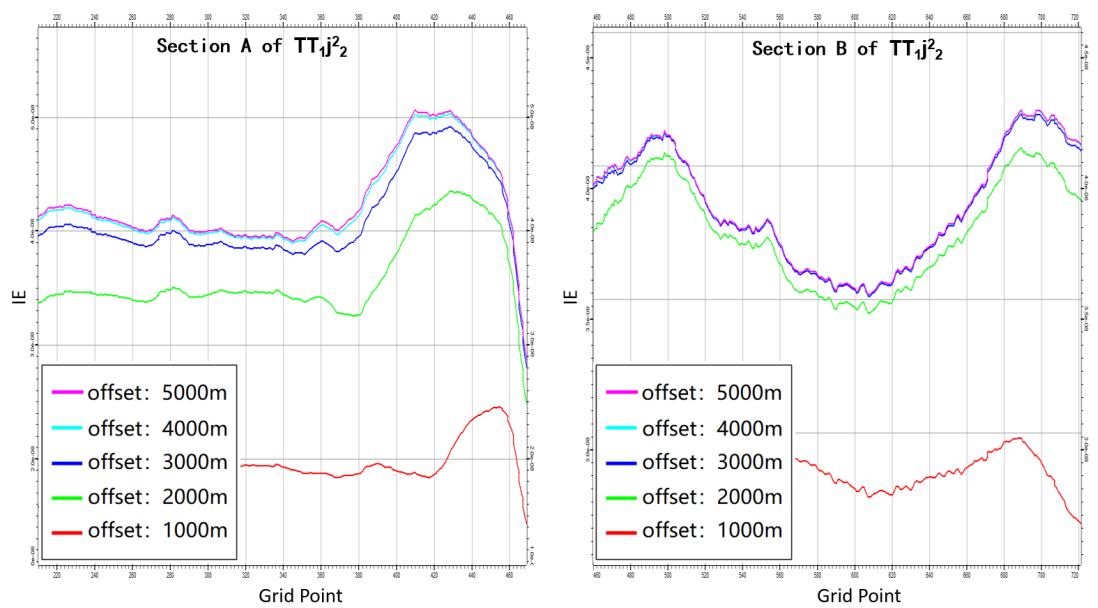

Figure 3. Comparative analysis of illumination curves with different offsets $\left(\mathrm{TT}_{1 j^{2}}{ }^{2}\right)$.
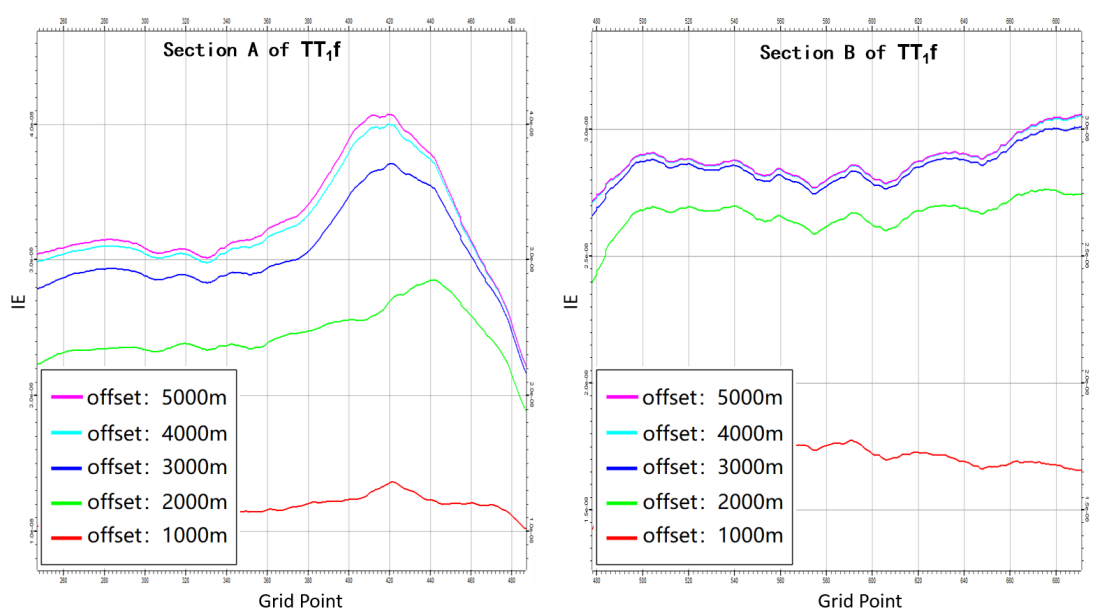

Figure 4. Comparative analysis of illumination curves with different offsets (TT $\left.\mathrm{T}_{1} \mathrm{f}\right)$.

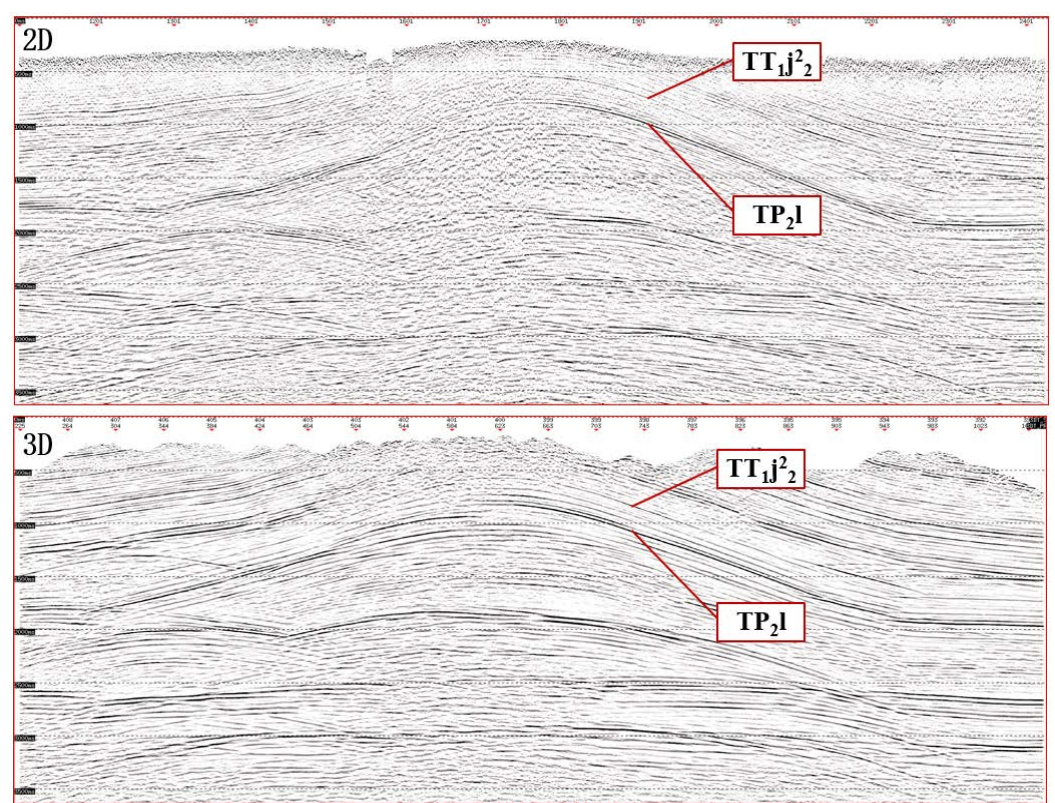

Figure 5. Comparison of new (3D) and old (2D) horizontal stack profiles. 
wide-azimuth, small charge in shallow well and high-sensitive single point geophone are adopted for acquisition. Among them, the maximum fold reached 144 times, the folds of the target layer $\mathrm{T}_{1} \mathrm{j}^{2}$ in the main part of the structure ranged from 19 to 24 times, and the aspect ratio of the target layer was 1 . The seismic data with high signal-to-noise ratio and high resolution are obtained by careful processing of fidelity and amplitude preservation.

Figure 5 is the comparison diagram of the $3 \mathrm{D}$ seismic profile and the $2 \mathrm{D}$ seismic profile at the same position as the $3 \mathrm{D}$ seismic. It can be seen that the $3 \mathrm{D}$ data has higher signal-to-noise ratio, better profile level, clearer imaging and more reasonable structural features.

\section{Conclusion}

The 3D acquisition design are mainly carried out according to the characteristics of the shallow buried depth of the target layer in HCX area, which effectively guarantees the imaging quality of the target layer of the seismic data. The high quality seismic data support the description of the structural details and fault distribution characteristics of the target layer, and improve the prediction accuracy of the thickness of the cap rock and bottom supporting layer, and meet the demand of fracture prediction and reservoir prediction.

\section{Acknowledgements}

This paper is a demonstration of some achievements of 3D seismic exploration project of HCX gas storage in Sichuan Basin. Thanks to all colleagues who participated in the project!

\section{Conflicts of Interest}

The authors declare no conflicts of interest regarding the publication of this paper.

\section{References}

Han, J. W., Zhang, W., Zhang, H., Xiao, H., \& Geng, C. (2020). The Application of WideAzimuth, Broadband, and High-Density Seismic Acquisition Technology in QLT area. SPG/SEG Nanjing 2020 International Geophysical Conference Proceedings, 163-166.

Li, W. W. (2008). Design of Seismic Observation System Based on Wave Equation forward Modeling. Oil Geophysical Prospecting, 43, No. 2, 134-144.

Guo, W. (2017). Method Research of 3D Seismic Geometry Evaluation and Design in Eastern Exploration Area. Master's Thesis, Beijing: China University of Geosciences.

Zhang, J. H. (2017). Discussion on the Design of Observation System in 3D Seismic Data Acquisition. Chinese Journal of Engineering Geophysics, 42, No. 6, 185-199.

Zhang, W. C. (2017). The Study of Optimal Design of 3D Seismic Observation System-D Basin of the Actual Data as an Example. Master's Thesis, Chengdu: Chengdu University of Technology. 